\title{
Comparing Four Sub-pixel Algorithms in MODIS Snow Mapping
}

\author{
Hongen Zhang \\ College of Environmental Science \\ and Engineering, Tongji University \\ 1239 Siping Road, Shanghai, \\ P.R.China, 200092
}

\author{
Jianfu Zhao \\ College of Environmental Science \\ and Engineering, Tongji University \\ 1239 Siping Road, Shanghai, \\ P.R.China, 200092
}

\author{
Shi Jiancheng \\ Institute for Computational \\ Earth System Sciences, \\ UCSB,USA
}

\begin{abstract}
Accurate monitoring of snow cover extent is an important research goal in the science of Earth systems. Now mixture modelling is important tool in the remote sensing community as researchers attempt to resolve sub-pixel, area information. In the paper, I tested four different sub-pixel analysis methods: Linear Mixture Model (LMM), Fuzzy c-means Clustering (FCM), Back-Propagation Neural Network (BPNN), and Support Vector Machine (SVM). Overall, the LMM and SVM method provided better estimates of the snow cover components than others, and the results of this study provide comprehensive information of the utility of sub-pixel analysis for the estimation of snow cover components and suggest that the comparatively accurate snow cover estimation is attainable from medium resolution satellite imagery.
\end{abstract}

\section{INTRODUCTION}

Snow is an important component of the Earth's surface. Because of its importance from both a scientific and resource management standpoint, accurate monitoring of snow cover extent is an important research goal in the geoscience. The high spatial resolution and numerous MODIS spectral bands in the 0.4 to $2.5 \mu \mathrm{m}$ wavelength region allow more accurate monitoring of snow cover extent on a global basis than is possible with other operational satellites.

Medium spatial resolution remotely sensed imagery is comparatively very cheap, but has a critical drawback "mixed" pixels. The problem leads to mis-classification of snow covered areas. Mixture modelling is becoming an increasingly important tool in the remote sensing community as researchers attempt to resolve the sub-pixel, mixture information, which arises from the overlapping land cover types within the pixel's instantaneous field of view.

0-7803-9050-4/05/\$20.00 @2005 IEEE.
In the paper, I tested four different sub-pixel analysis methods: Linear Mixture Model (LMM), Fuzzy c-means Clustering (FCM), Back-Propagation Neural Network (BPNN), and Support Vector Machine (SVM). These results are significant in that they demonstrate that medium resolution remotely sensed imagery such as Modis provide a cost effective image data source for snow monitoring.

\section{THOERETICAL BACKGROUND}

\section{A. Linear Mixture Model}

Linear spectral mixture model can be describe:

$$
\mathbf{A x}=\mathbf{b} \text {. }
$$

Where $\mathbf{A} \in R^{m \times n}, \mathbf{x} \in R^{n}, \mathbf{b} \in R^{m}, m>n, \mathbf{b}$ denote the $m \times 1$ image pixel and $\mathbf{A}$ is the $m \times n$ image matrix, each columns of $\mathbf{A}$ is an endmember spectrum for a specific endmember.

There exist many different linear spectral unmixing approaches, and most of them are derived from linear least squares approaches. Linear least squares estimation produces a concentration vector $\mathbf{x}_{\mathbf{L S}}$ and a perturbation vector $\mathbf{r}$ subject to the following conditions:

$$
\mathbf{A} \mathbf{x}_{L S}=\mathbf{b}+r \text { s.t. }\|r\|^{2} \text { minimized }
$$

$\|r\|^{2}$ denote the sum of squared components of the vector $r$.

The solution to this problem when $\mathbf{A}$ is of full column rank is:

$$
\mathbf{x}_{L S}=\left(\mathbf{A}^{t} \mathbf{A}^{-1}\right) \mathbf{A}^{t} \mathbf{b}
$$

\section{B. Fuzzy c-means Clustering}

Fuzzy c-means clustering(FCM) is the best known and the most popular fuzzy clustering method. FCM measures the fuzzy membership value of data for each cluster based on the distance between the cluster center and the data in the feature 
space of remotely sensed imagery. The fuzzy membership value makes FCM more flexible and useful in practical applications, such as sub-pixel analysis[2]. The performance of FCM has been shown to be better approach than the hard c-mean clustering method(Chi et al.1996).

The FCM is based on minimization of the following objective function to $U$, a fuzzy c-partition of the data set, and to $V$,a set of $K$ prototype:

$$
J_{m}(U, V)=\sum_{j=1}^{n} \sum_{i=1}^{c}\left(u_{i j}\right)^{m}\left\|X_{j}-V_{i}\right\|^{2}, 1 \leq m<\infty
$$

Where $\mathrm{m}$ is any real number greater than $1, U_{i j}$ is the degree of member of $X_{j}$ in the cluster $i, X_{j}$ is the $j$ th of d-dimensional measured data, $V_{i}$ is the dimension of the cluster.

Fuzzy partition is carried out through an iterative of (3) with the update of membership uij and the cluster centers $V_{i}$ by

$$
\begin{aligned}
& u_{i j}=1 / \sum_{k=1}^{c}\left(\frac{d_{i j}}{d_{i k}}\right)^{\frac{2}{m-1}} \\
& v_{i}=\frac{\sum_{k=1}^{n}\left(u_{i j}\right)^{m} x_{j}}{\sum_{j=1}^{n}\left(u_{i j}\right)^{m}}, 1 \leq i \leq c
\end{aligned}
$$

The criteria in this iteration will stop when $\max _{i j}\left[\left|u_{i k}-\hat{u}_{i k}\right|\right]<\varepsilon$, where $\varepsilon$ is a termination criteria between 0 and 1.

\section{Back-Propagation Neural Network}

Since the mid 1980's ,artificial neural networks have been increasingly applied to diverse remote sensing analyses to overcome the limitations of traditional statistical methods and

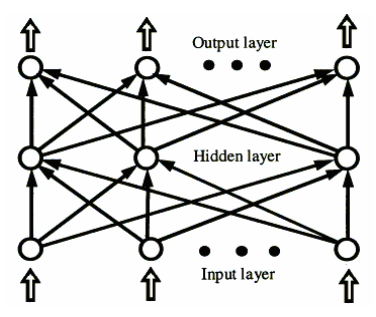

Figure 1. BP neural network

to fully utilize the potential of newly deployed satellite sensors. It has been applied on sub-pixel classification.[3]

Back-propagation is a supervised learning algorithm that applies to non-linear, multilayer, feed-forward structure of nodes (networks). The architecture of a BP network refers to the way it decodes information, that is the direction of information during recall. In a BP neural network the nodes are organized in input, hidden, and output layers, as Figure 1.

\section{Support Vector Machine}

The principle of the SVM-based solution for the learning process is very briefly described below[4]. Suppose that the training data:

$\mathrm{D}=\left\{\left(\mathbf{x}_{1}, y_{1}\right), \cdots,\left(\mathbf{x}_{l}, y_{l}\right)\right\}, \quad \mathbf{x} \in R^{n}, \quad y \in\{-1,1\}(6)$

can be separated by a hyperplane:

$$
w^{T} x+b=0
$$

The set of vectors is said to be optimally separated by the hyperplane if it is separated without error and the distance between the closest vector to the hyperplane is maximal.

A separating hyperplane in canonical form must satisfy the following constraints,

$$
\left.y_{i}\left[w^{T} x_{i}+b\right] \geq 1\right)
$$

Then the hyperplane that optimally separates the data is the one that minimises

$$
\Phi(w)=\frac{1}{2}\|w\|^{2}
$$

Classical Lagrangian duality enables the problem. The optimal separating hyperplane is given by

$$
\begin{aligned}
& w^{*}=\sum_{i=1}^{l} \alpha_{i} y_{i} x_{i} \\
& b^{*}=-\frac{1}{2} w^{*^{T}}\left(x_{r}+x_{s}\right)
\end{aligned}
$$

where $x_{r}$ and $x_{s}$ are any support vector from each class satisfying,

$$
\alpha_{r}, \alpha_{s}>0, \quad y_{r}=-1, \quad y_{s}=1
$$

The hard classifier is then,

$$
f(x)=\operatorname{sgn}\left(w^{* T} x+b^{*}\right)
$$

A soft classifier is used here which linearly interpolates the margin,

$$
f(x)=h\left(w^{* T}+b\right)
$$

where, $h(z)=\left\{\begin{array}{lcc}0 & : & z<-1 \\ (z+1) & / 2:-1 \leq z \leq 1 \\ 1 \quad: & z>1\end{array}\right.$

III. DATA and METHODS 


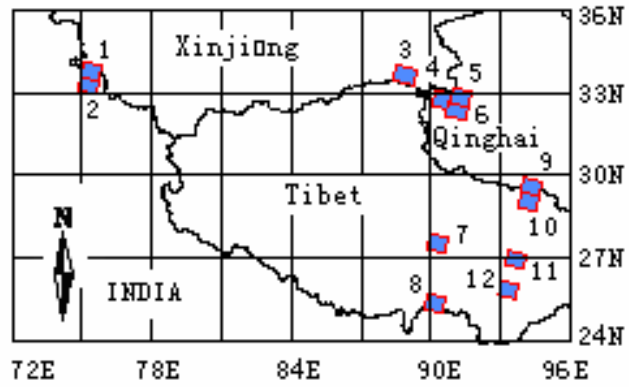

Figure 2. Location of Aster image

Aster provides high spatial resolution (15-90 m) multispectral images of the Earths surface. These data bridge the gap between field observations and data acquired by coarse spatial resolution instruments such as MODIS,and between local-process models and regional models.

Here we classify ASTER image in three visible and near-infrared channels with 15-m resolution as "ground truth" to validate our MODIS snow mapping algorithm.

The study area is situated in the Tibetan Plateau. The available data consist of 12 MODIS image and Aster image corresponding days and area for validation, as shown in figure 2 .

Selecting endmembers is key in linear mixture model, here endmembers is determined by averaging the pixel value image 5(fig.2) as an example is shown in figure 3.

In FCM, the fuzzy membership value is not linearly correlated with the class proportion. To utilize the fuzzy membershipvaue to estimate the actual composition of snow in a mixed pixel, simulated data is created through mixing the endmenbers spectral value. The simulated data and the image data is applied together in FCM, and the relation is found.

The simulated data is also took as training data to establish the networks in BP neural networks algorithm. The parameter in BP is set up by testing again and again.

The training data in SVM is selected manually from image, the data is split into two base class: snow and non-snow (soil, grass, shrubs, etc), image 5(fig.2) as an example is shown in figure 3.

\section{RESULT and CONCLUTION}

The result of unmixing for snow is shown in table in TABLE I . In FCM, only the pixels that the percent of snow cover area in it is greater than $15 \%$ are count,because previous work showed lower accuracy when the proportion is less than $15 \%$.

The error of SVM is less than $4.4 \%$ as compared to the reference data from Aster image classification and the average

TABLE I UNMIXING RESULT of LMM, BPNN,SVM and FCM

\begin{tabular}{|c|c|c|c|c|c|c|c|c|c|c|}
\hline \multirow[b]{2}{*}{ NO. } & \multirow{2}{*}{$\frac{\text { Aster Image }}{\text { Area }(\mathrm{Km} 2)}$} & \multicolumn{2}{|c|}{ LMM } & \multicolumn{2}{|c|}{ BPNN } & \multicolumn{2}{|c|}{ SVM } & \multirow{2}{*}{$\frac{\operatorname{LMM}(>15 \%)}{\text { Area }(\mathrm{Km} 2)}$} & \multirow{2}{*}{$\frac{\operatorname{FCM}(>15 \%)}{\text { Area }(\mathrm{Km} 2)}$} & \multirow{2}{*}{$\frac{\text { FCM vs. LMA }}{\text { Error(\%) }}$} \\
\hline & & $\operatorname{Area}\left(\mathrm{Km}^{2}\right)$ & Error(\%) & $\operatorname{Area}\left(\mathrm{Km}^{2}\right)$ & Error(\%) & $\operatorname{Area}\left(\mathrm{Km}^{2}\right) \mathrm{a}$ & Error(\%) & & & \\
\hline No.1 & 580.973 & 581.767 & 0.1366 & 558.693 & -3.835 & 584.763 & -3.8 & 516.516 & 519.326 & 0.5438 \\
\hline No.2 & 794.51 & 816.193 & 2.7291 & 813.484 & 2.3881 & 790.382 & 2.39 & 789.724 & 790.537 & 0.1029 \\
\hline No.3 & 607.591 & 627.412 & 3.2622 & 616.594 & 1.4817 & 629.622 & 1.48 & 604.658 & 604.80 & 0.0239 \\
\hline No.4 & 301.392 & 295.402 & -1.987 & 303.79 & 0.7959 & 285.327 & 0.8 & 114.416 & 113.895 & -0.455 \\
\hline No. 5 & 592.745 & 597.395 & 0.7846 & 618.983 & 4.4266 & 612.598 & 4.43 & 583.440 & 580.972 & -0.423 \\
\hline No.6 & 146.785 & 151.542 & 3.241 & 150.908 & 2.8087 & 142.346 & 2.81 & 123.875 & 123.904 & 0.0231 \\
\hline No.7 & 512.91 & 525.762 & 2.5055 & 533.928 & 4.0975 & 519.83 & 4.1 & 504.366 & 503.691 & -0.134 \\
\hline No.8 & 346.45 & 343.891 & -0.739 & 364.21 & 5.1258 & 343.92 & 5.13 & 359.915 & 356.754 & -0.878 \\
\hline No.9 & 383.91 & 380.512 & -0.884 & 370.313 & -3.54 & 383.09 & -3.5 & 326.733 & 326.88 & 0.0458 \\
\hline No.10 & 1015.1 & 993.112 & -2.17 & 973.68 & -4.085 & 1005.2 & -4.1 & 960.585 & 959.232 & -0.141 \\
\hline No.11 & 749.87 & 738.635 & -1.498 & 731.981 & -2.386 & 759.84 & -2.4 & 724.375 & 723.487 & -0.123 \\
\hline No. 12 & 961.2 & 943.942 & -1.796 & 960.829 & -0.039 & 943.64 & -0.04 & 961.2 & 941.605 & -2.080 \\
\hline
\end{tabular}

selected by scatter plot of band 4 and band 6 of MODIS data,

of error is $1.9 \%$, the least error just is $0.004 \%$. The error of 
LMM is less than $3.26 \%$ compared to the reference data from Aster image classification and the average of error is $5.1 \%$, the least error is $0.14 \%$. The error of $\mathrm{BPNN}$ is less than $3.26 \%$ ompared to the reference data from Aster image classification and the average of error is $3.4 \%$, the least error is $0.79 \%$. The relative error between FCM and LMM is less than $2.08 \%$, and most of them is less than $0.5 \%$. In summary, all of the four sub-pixel algorithms for MODIS snow mapping described previously provided considerable accuracy of snow cover area, and LMM and SVM method provided the best estimates of the snow cover components.

The linear mixture assumption of the endmembers spectra of LMM is upheld to a large extent as evidenced by the rather high accuracy of snow cover estimation. The results of this study provide comprehensive information of the utility of sub-pixel analysis for the estimation of snow cover components and suggest that the comparatively accurate snow cover estimation is attainable from medium resolution satellite imagery. These results are significant in that they demonstrate that medium resolution remotely sensed imagery such as Modis provide a cost effective image data source for snow monitoring.

\section{REFERENCE}

[1]Adams J. B., Sabol D. E.,Kapos V..Classification of multispectral images based on fractions of endmembers: application to land-cover in the Brazilian Amazon. Remote Sens. Environ.1995,52:137-154.

[2]Foody G M, Cox D P. Sub-pixel land cover composition using a linear mixture model and fuzzy membership functions. International Journal of Remote Sensing, 1994,15: 619-631

[3]Carpenter G., Gopal S., Macomber M. S., Woodcock C.. A neural network method for mixture estimation for vegetation mapping, Remote Sensing of Environment,70:138-152, 1999

[4]Steve R. Gunn .Support Vector Machines for Classification and Regression,,17-30,1998

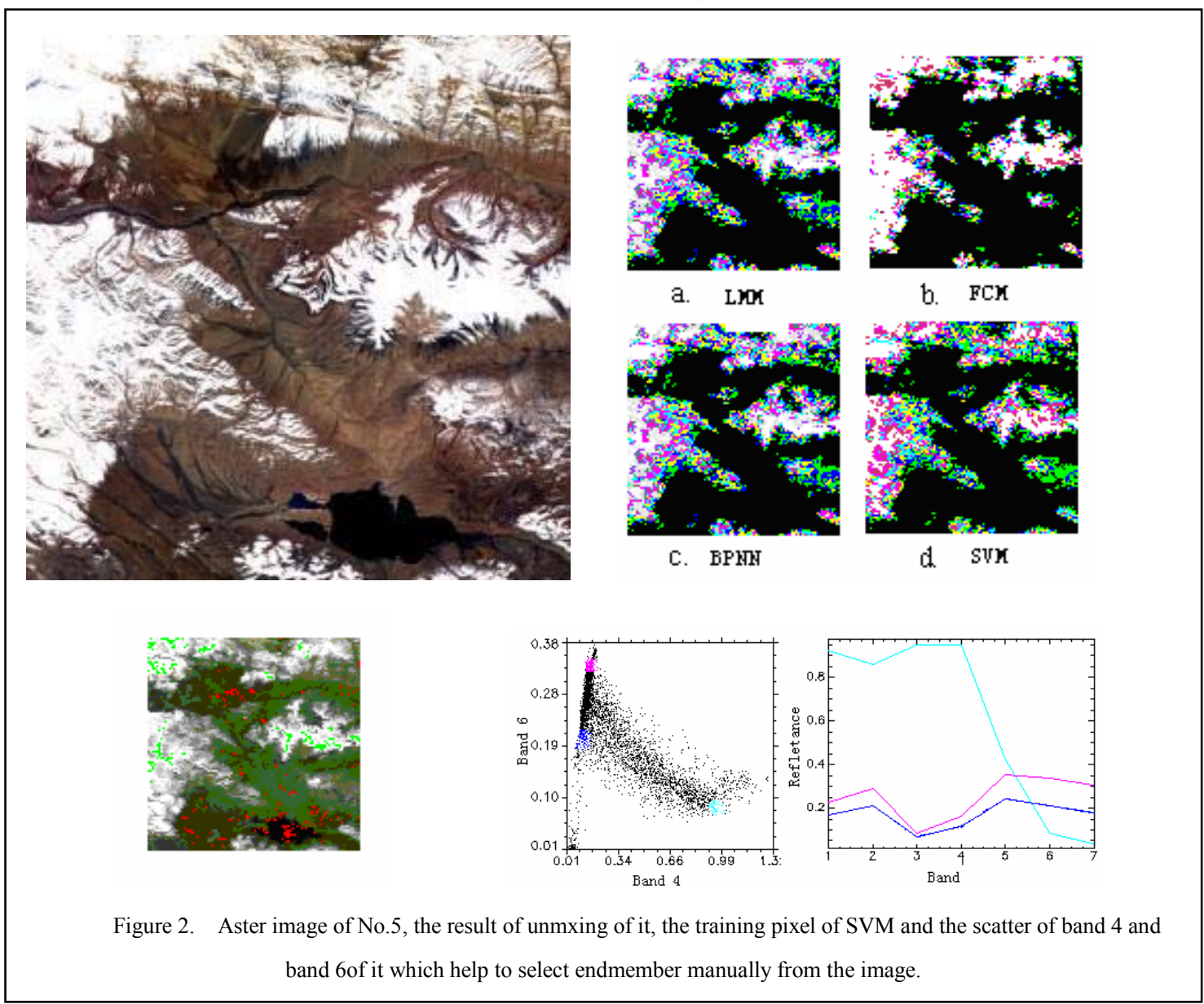

